\section{References}

1. Weber DJ, Al-Tawfiq JA, Babcock HM, et al. Multisociety statement on COVID-19 vaccination as a condition of employment for healthcare personnel. Infect Control Hosp Epidemiol 2021. doi: 10.1017/ice.2021.322.

2. Schaffzin J, Shenoy E. Viewpoint: COVID-19 vaccines should be a condition of employment for healthcare personnel. Becker's Hospital Review website. https://www.beckershospitalreview.com/workforce/viewpoint-covid-19vaccines-should-be-a-condition-of-employment-for-healthcare-personnel. html. Published July 14, 2021. Accessed August 6, 2021.

3. Joint statement in support of COVID-19 vaccine mandates for all workers in health and long-term care. American College of Physicians (ACP) website. https://www.acponline.org/acp_policy/statements/ joint_statement_covid_vaccine_mandate_2021.pdf. Published July 26, 2021. Accessed August 8, 2021.

4. VHA directive 1193(1): coronavirus disease 2019 vaccination program for Title 38 healthcare personnel. Department of Veterans' Affairs website. https://www.va.gov/vhapublications/ViewPublication.asp?pub_ID=9355. Amended August 5, 2021. Accessed August 8, 2021.

5. Hanrahan JA, Kammeyer JA, Sievert D, Naylor B, Khuder S, Kaminski B. Implementation of Society for Hospital Epidemiology of America (SHEA) and Centers for Disease Control and Prevention (CDC) Outbreak Response Training Program tools to develop a dedicated coronavirus disease 2019 (COVID-19) hospital in Lucas County, Ohio. Infect Control Hosp Epidemiol 2021. doi: 10.1017/ice.2021.158.

\title{
Severe acute respiratory coronavirus virus 2 (SARS-CoV-2) delta variant of concern breakthrough infections: Are vaccines failing us?
}

\author{
Ali Nowroozi MD² (1) and Nima Rezaei MD, $\mathrm{PhD}^{2,3,4}$ (1) \\ ${ }^{1}$ School of Medicine, Tehran University of Medical Sciences, Tehran, Iran, ${ }^{2}$ Network of Immunity in Infection, Malignancy and Autoimmunity (NIIMA), Universal \\ Scientific Education and Research Network (USERN), Tehran, Iran, ${ }^{3}$ Research Center for Immunodeficiencies, Children's Medical Center, Tehran University of \\ Medical Sciences, Tehran, Iran and ${ }^{4}$ Department of Immunology, School of Medicine, Tehran University of Medical Sciences, Tehran, Iran
}

To the Editor-The severe acute respiratory coronavirus virus 2 (SARS-CoV-2) delta (formerly B.1.617.2) variant of concern (VOC) has become or is becoming the most prevalent type of SARS-CoV-2 in many countries. ${ }^{1}$ Whether available vaccines can protect people against this variant as effectively as against other common variants is yet unknown.

Most countries have already started public vaccination against coronavirus disease 2019 (COVID-19), and the share of people who have received at least 1 vaccine dose reaches as high as $70 \%$ in some regions. ${ }^{2}$ The effect of vaccination is evident in the pattern of daily new cases and deaths in countries with high vaccination rates. ${ }^{3}$ However, many of these nations are experiencing a surge in COVID-19 cases $^{3}$ possibly attributable to novel SARSCoV-2 variants, especially the delta VOC. On the other hand, number of deaths has not increased significantly, ${ }^{3}$ which might be due to the effect of vaccines against serious illness or the relatively short time since the dominance of the new variant. Whether currently available vaccines stand the test of time and limit the COVID-19 burden on communities remains to be seen.

Although several studies have indicated reduced neutralizing antibody activity (ie, vaccine effectiveness) against the delta VOC compared with the B.1.17 lineage and the wild-type virus, ${ }^{4}$ only 2 Medline-indexed studies ${ }^{5,6}$ have assessed vaccine efficacy against this variant. Using data from registries in Scotland, Sheikh et $\mathrm{al}^{5}$ estimated vaccine efficacy against symptomatic delta VOC infection 14 days after the second dose for BNT162b2 (Pfizer-BioNTech) and ChAdOx1 (Oxford-AstraZeneca) at 83\% and $61 \%$, respectively. Efficacy regardless of symptom presence was $79 \%$ for BNT162b2 and $60 \%$ for ChAdOx1. Another study

\footnotetext{
Author for correspondence: Prof. Nima Rezaei, E-mail: rezaei_nima@yahoo.com Cite this article: Nowroozi A and Rezaei N. (2022). Severe acute respiratory coronavirus virus 2 (SARS-CoV-2) delta variant of concern breakthrough infections: Are vaccines failing us?. Infection Control \& Hospital Epidemiology, 43: 1754-1755, https://doi.org/10.1017/ice.2021.363
}

in the United Kingdom ${ }^{6}$ indicated $67.0 \%$ efficacy for ChAdOx 1 and $88.0 \%$ for BNT162b2 vaccines against symptomatic delta VOC infection, 2 weeks after the second injection. Vaccine efficacy 21 days after the first dose was low for both ChAdOxl and BNT162b2 (30.0\% and 35.6\%, respectively).

Although these are the only published studies, promising results have also been found in unpublished investigations. A preprint study by Nasreen et $\mathrm{al}^{7}$ conducted in Canada, showed $56 \%, 72 \%$, and $67 \%$ efficacy against symptomatic delta VOC infection for BNT162b2, mRNA-1273 (Moderna), and ChAdOx1, respectively, 2 weeks after the first dose, and $87 \%$ efficacy 7 days after the second dose of BNT162b2. The vaccines demonstrated $78 \%-96 \%$ protection against serious illness, 14 days after their first dose, which will most likely be even higher after full vaccination.

Most healthcare personnel in Iran have been vaccinated with either ChAdOx1, Gam-COVID-Vac (Sputnik V, Gamaleya), BBIBP-CorV (Sinopharm), or BBV152 (Bharat) vaccine. However, as the country experiences the fifth wave of COVID- $19,{ }^{3}$ there are many reports of infection, serious respiratory involvement, hospitalization, and even death among vaccinated persons. ${ }^{8}$ As indicated in phase 3 trials, ${ }^{9}$ none of the currently available vaccines have $100 \%$ protection against infection, and community protection against serious illness is expected to differ from those of clinical trials. The incidence and severity of breakthrough infections cases is concerning and raises doubts regarding the efficacy of the vaccines utilized against the delta VOC. The proper way of addressing this issue is by conducting studies to assess the efficacy of vaccines against this variant and other VOCs. Unfortunately, to the best of our knowledge, no published or preprint studies have investigated the efficacy of the 4 mentioned vaccines against the delta VOC, except for ChAdOx1. Nonetheless, vaccine producers claim that their vaccines are effective to some degree against the mentioned variant (eg, 90\% for Gam-COVID-Vac and $65.2 \%$ for BBV152). ${ }^{10,11}$

We encourage the scientific community to further evaluate the vaccines that have limited data on their effectiveness (eg, 
those mentioned in this letter) by conducting randomized clinical trials and assessing protection against infection, rather than the ability to produce neutralizing antibodies because the former reflects the real-world effects of the vaccine more accurately. Governments and healthcare organizations must implement registry systems for breakthrough infections, to better understand the ongoing pandemic and outcomes of vaccination in the community. Finally, vaccines might be less effective against novel SARS-CoV-2 variants. Research institutes and vaccine producers should be prepared for such conditions and should develop new strategies to increase effectiveness against resistant lineages, such as introducing additional booster doses or reformulating the vaccines.

Acknowledgments.

Financial support. No financial support was provided relevant to this article.

Conflicts of interest. All authors report no conflicts of interest relevant to this article.

\section{References}

1. Share of COVID sequences that are the delta variant. Our World in Data website. https://ourworldindata.org/grapher/covid-cases-delta. Accessed July 20, 2021.

2. Share of people who received at least one dose of COVID-19 vaccine. Our World in Data website. https://ourworldindata.org/grapher/share-peoplevaccinated-covid?tab=table. Accessed July 20, 2021.
3. Daily new confirmed COVID-19 cases and deaths. Our World in Data website. https://ourworldindata.org/grapher/daily-covid-cases-deaths-7day-ra. Accessed July 20, 2021.

4. Hoffmann M, Hofmann-Winkler H, Krüger N, et al. SARS-CoV-2 variant B.1.617 is resistant to bamlanivimab and evades antibodies induced by infection and vaccination. Cell Rep 2021. doi: 10.1016/j.celrep.2021.109415.

5. Sheikh A, McMenamin J, Taylor R, Robertson C, Public Health Scotland and the EAVE II Collaborators. SARS-CoV-2 delta VOC in Scotland: demographics, risk of hospital admission, and vaccine effectiveness. Lancet 2021;397:2461-2462.

6. Lopez-Bernal J, Andrews N, Gower C, et al. Effectiveness of COVID-19 vaccines against the B.1.617.2 variant. medRxiv 2021. doi: 10.1101/2021.05.22. 21257658.

7. Nasreen $\mathrm{S}$, He S, Chung H, et al. Effectiveness of COVID-19 vaccines against variants of concern, Canada. medRxiv 2021. doi: 10.1101/2021.06.28. 21259420.

8. Independent Persian. ابتلاى كادر درمان به كرونا بِ از دريافت واكسن 2021 Available from: https://www.independentpersian.com/node/163041/. Accessed July 20, 2021.

9. Cheng H, Peng Z, Luo W, et al. Efficacy and safety of COVID-19 vaccines in phase III trials: a meta-analysis. Vaccines (Basel) 2021;9:582.

10. India's Bharat Biotech says vaccine is effective against delta variant. Wall Street Journal website. https://www.wsj.com/articles/indias-bharat-biotech-saysvaccine-is-effective-against-delta-variant-11625325879. Accessed July 20, 2021.

11. Russia's Sputnik V shot around $90 \%$ effective against delta variant, developers say. Reuters website. https://www.reuters.com/business/healthcarepharmaceuticals/russias-sputnik-v-vaccine-less-effective-against-deltavariant-tass-2021-06-29/. Accessed July 20, 2021.

\title{
SARS-CoV-2 vaccine breakthrough infections among healthcare workers in a large Belgian hospital network
}

\author{
Dieter Geysels $M D^{1}$ (1), Pierre Van Damme MD, prof. ${ }^{2}$, Walter Verstrepen PharmD, $\mathrm{PhD}^{1}$, Peggy Bruynseels $\mathrm{MD}^{1,3}$, \\ Bea Janssens $M D^{4}$, Patrick Smits $M D^{5}$ and Reinout Naesens MD ${ }^{1,3}$ \\ ${ }^{1}$ Department of Medical Microbiology, ZiekenhuisNetwerk Antwerpen, B-2020 Antwerp, Belgium, ${ }^{2}$ Centre for the Evaluation of Vaccination, Vaccine and Infectious \\ Disease Institute, University of Antwerp, B-2610 Wilrijk, Belgium, ${ }^{3}$ Department of Infection Prevention and Control, ZiekenhuisNetwerk Antwerpen, B-2020 \\ Antwerp, Belgium, ${ }^{4}$ IDEWE, External Service for Prevention and Protection at Work, B-2000 Antwerp, Belgium and ${ }^{5}$ Agency for Care and Health, Prevention \\ Division, Flanders, B-2018 Antwerp, Belgium
}

To the Editor-Since the first occurrence of SARS-CoV-2, different vaccines have been produced, tested, and approved in record time. However, the exact vaccination effect has yet to be determined in the ever-changing landscape of SARS-CoV-2 variants. ${ }^{1,2}$

\section{Breakthrough infections}

As of June 15, 2021, the overall vaccination rate in Belgium was $62.8 \%$ having received 1 dose (adult population), of whom $35.2 \%$ had fully completed their vaccination scheme. ${ }^{3}$ Belgian healthcare workers (HCWs) have a high exposure to COVID-19: the estimated percentage of confirmed Belgian COVID-19 cases is significantly higher among those working in healthcare facilities than among employees in other industries as well as the national average. ${ }^{4}$

Author for correspondence: Dr. Geysels Dieter, E-mail: dieter.geysels@zna.be

Cite this article: Geysels D, et al. (2022). SARS-CoV-2 vaccine breakthrough infections among healthcare workers in a large Belgian hospital network. Infection Control \& Hospital Epidemiology, 43: 1755-1757, https://doi.org/10.1017/ice.2021.326
Because HCWs were prioritized in the vaccination strategy, studying this population provided early data with which to analyze its effect. Vaccination in Belgium is not mandatory.

Vaccination of HCWs in ZNA started on January 18, 2021. ZNA is a 2,500-bed, public, multiple-site, hospital network in the Antwerp region. It comprises 3 acute-care hospitals, a children's hospital, and 5 chronic care facilities. Depending on their availability, 3 different vaccines were used: BNT162b2 (Comirnaty, BioNTech/Pfizer, Mainz, Germany), mRNA-1273 (COVID-19 Vaccine Moderna, Moderna, Cambridge, MA) and AZD1222 (Vaxzevria, Astra Zeneca, Cambridge, UK). The impact of vaccinations on the positive test ratio was evaluated from March 1 through April 30, 2021, a period with continuing and substantial viral circulation in the Belgian population. ${ }^{3}$ Tests were performed for contact tracing or COVID-like symptoms. Among 3,491 fully vaccinated ZNA HCWs, 9 (0.3\%) tested positive for SARS-CoV-2 (RT-PCR, Cobas 6800, Roche). After excluding 1 case, following CDC guidelines on persistent shedding, ${ }^{5} 22(1.0 \%)$ of 2,215 unvaccinated HCWs $(n=584)$ or partially vaccinated HCWs $(n=1,631)$ tested 